\title{
ARTICLE
}

\section{A new spent fuel source characterization code CHARS and its application to the shielding of the thorium cycle}

\author{
Baptiste Leniau* and Jonathan N. Wilson \\ Institut de Physique Nucléaire, 15 rue Georges Clemenceau, Orsay, 91406, FRANCE
}

\begin{abstract}
Radioprotection measures needed in the nuclear fuel cycle require accurate knowledge of the radioactive sources involved. For innovative nuclear reactors such as Generation IV designs radiation sources (alpha, beta, gamma and neutron) in the spent fuel need to be calculated in order to understand the radioprotection needed in all aspects of the fuel cycle (transport, reprocessing, fuel fabrication, waste storage, etc.). For this purpose we have developed CHARS a set of source characterization tools coupled to our code MURE (MCNP Utilities for Reactors Evolution) which is a precision research code for fuel evolution. MURE determines inventories of around 800 nuclei during irradiation and cooling via a series of MCNP5 calculations and numerical integration of Bateman's equations. With the CHARS package using the ENSDF libraries, it's possible to generate alpha, beta and gamma spectra of the nuclear fuel at the end of cycle from any given reactor design. These complex source definitions are then used to generate automatic MCNP5 inputs for radioprotection calculations, allowing us to undertake a wide range of radioprotection studies. The first use of these tools was to estimate additional shielding in the French fuel cycle in case of switching from the current uranium $(\mathrm{U} / \mathrm{Pu})$ cycle to the thorium $(\mathrm{Th} / \mathrm{U})$ cycle. Irradiated thorium-based fuels produce small quantities of ${ }^{232} \mathrm{U}$, which has a relatively short half-life (69 years) and emits a hard gamma of 2.6 $\mathrm{MeV}$ at the end of its decay chain. ${ }^{232} \mathrm{U}$ is synthesized in mainly two ways: ${ }^{233} \mathrm{U}(\mathrm{n}, 2 \mathrm{n})$ and ${ }^{232} \mathrm{Th}(\mathrm{n}, 2 \mathrm{n})$ followed by ${ }^{231} \mathrm{~Pa}(\mathrm{n}, \gamma)$. From the results of radioprotection calculations we estimate additional thickness of shielding required for the back end of the fuel cycle and show that the greatest constraints occur for the fuel manufacturing. On the other hand the neutron yield for some thorium-based fuels will be lower in $\mathrm{Th} / \mathrm{U}$ than in $\mathrm{U} / \mathrm{Pu}$ cycle and as a result necessary neutronic shielding will be reduced.
\end{abstract}

Keywords: spent fuel; thorium cycle; gamma spectra; neutron spectra; Monte Carlo; MCNP; shielding; dose rate; MURE

\section{Introduction}

The next generation of nuclear reactors (Generation IV) is currently being developed and is intended to improve fissile resource utilization, reduce waste production, increase safety, and enhance anti-proliferation. The future use of Generation IV technologies requires detailed designs, not just of the reactors themselves, but their entire nuclear fuel cycle. Correct dimensions for the factories of the back end of the fuel cycle (fuel reprocessing, re-fabrication, transportation and waste disposal) need to be calculated along with the design of the radioprotection shielding needed. This requires accurate knowledge of the radioactive sources (alpha, beta, gamma and neutron) involved at each stage in the fuel cycle. In order to facilitate such calculations, we developed a code for source characterization (CHARS) coupled with the existing precise fuel depletion code [1] (MURE), which could produce accurate spent fuel

*Corresponding author. Email: leniau@ipno.in2p3.fr composition information for complex reactor geometries using different fuel cycles.

\section{Tools used and developed}

\subsection{MURE}

MURE is an interactive precision research code coupled with widely used Monte-Carlo based neutron, photon, electron, or coupled neutron/photon/electron transport code, MCNP [2]. MURE determines inventories of around 800 nuclei during fuel irradiation and cooling via a series of MCNP5 calculations and numerical integration of the set of coupled differential equations that govern their production and destruction (Bateman's equations). MURE is also a $\mathrm{C}++$ interface for MCNP5 facilitating automated input/output, the geometry definition and post-processing of results. A graphical user interface (MureGui) allows access to the data generated during the fuel evolution calculation: $\mathrm{k}_{\mathrm{eff}}$, fluxes, decay heats, inventories, cross-sections, radio toxicities, reaction 
rates. In addition the MureGui now computes radiation spectra using the CHARS package.

\subsection{CHARS (CHaracterization of Radioactive Sources)}

CHARS uses the results of MURE's precision fuel depletion calculation and combines them with the best available nuclear decay data to reconstruct neutron, gamma, alpha and beta spectra of any spent fuel after irradiation. This includes during the cooling phase in-core, and before and after reprocessing out of core.

\subsubsection{Gamma spectra}

The ability to read nuclear structure data in ENSDF [3] format and the knowledge of nuclei inventories during cooling allows reconstruction of gamma spectra from the decays of all nuclei combined together. Gammas taken into account are gammas from $\alpha, \beta^{-}, \beta^{+}$, electron capture and isomeric transition decay processes. $\mathrm{X}$ rays originating from bremsstrahlung of $\beta^{-}$can be estimated using MCNP and beta spectra calculated using a simple Fermi theory where all the transitions are allowed (the details of this calculation are not discussed here since it is not important for fabrication of new fuel). The nuclear structure data used comes from [4] but it is possible to use any nuclear structure data library in the ENSDF format.

\subsubsection{Neutron spectra}

Neutrons emitted from the spent fuel are produced by two major processes, one is spontaneous fission and another is $(\alpha, n)$ reactions on light nuclei. In the CHARS code the neutron spectra from spontaneous fission is computed using a Watt distribution W(E).

$$
W(E)=C \exp \left(-\frac{E}{a}\right) \cdot \sinh (\sqrt{b \cdot E})
$$

Where $\mathrm{a}$ and $\mathrm{b}$ are coefficients depending on the nucleus and $\mathrm{C}$ the normalization constant. The neutron yield of the nucleus by spontaneous fission at energy $\mathrm{E}$ is:

$$
A(E)=\lambda N<v>B R \cdot W(E)
$$

Where BR is the branching ratio of the spontaneous fission and $\langle v\rangle$ the average number of neutron per fission. Values of BR are from ENSDF, $<v>$ from ENDF 7.1 and $a, b$ are from a fit performed by Madland (see [5]). $\lambda$ is the decay constant and $\mathrm{N}$ is the number of nuclei. Neutrons are also produced by $(\alpha, n)$ reactions on light nuclei during slowing down of alpha particle in the spent fuel. Two principal reactions ${ }^{17} \mathrm{O}(\alpha, n){ }^{20} \mathrm{Ne}$ and ${ }^{18} \mathrm{O}(\alpha, n){ }^{21} \mathrm{Ne}$ are taken into account for calculation of neutron yield attributed from $(\alpha, n)$ reactions. To compute neutron spectra from these reactions, one needs alpha spectra (calculated using ENSDF data [4]), stopping power in the media, cross sections leading to different level of ${ }^{20} \mathrm{Ne}$ and ${ }^{21} \mathrm{Ne}$ and oxygen density. The stopping powers are calculated for $\mathrm{UO}_{2}$ media using the SRIM (Stopping and Range of Ions in Matter) [6] code and the total $(\alpha, n)$ cross-section $\left(\sigma_{\text {tot }}\right)$ is taken from JENDL/AN-2005 [7]. The ratio $\frac{\sigma_{l}(E)}{\sigma_{l(E)}}$, where $\sigma_{l}$ is the $(\alpha, n)$ cross section of the reaction leading to the level 1 of the daughter nucleus, is calculated with the nuclear reaction simulation code TALYS [8]. Thick target, homogenous mixture and isotropic emission of neutrons in the center of mass are assumed for the calculation.

\subsection{Validation}

\subsubsection{Benchmark with CESAR 5.33}

CESAR is a qualified depletion code developed by the CEA and AREVA (COGEMA) [9]. The benchmark consists of calculating the gamma spectrum of a standard pressurized water reactor (PWR) 17x17 uranium oxide (UOX) assembly, enriched to $5 \%$ mol. ${ }^{235} \mathrm{U}$ at a fuel burn-up of $55 \mathrm{GWd} / \mathrm{tHM}$ and various different fuel cooling times.

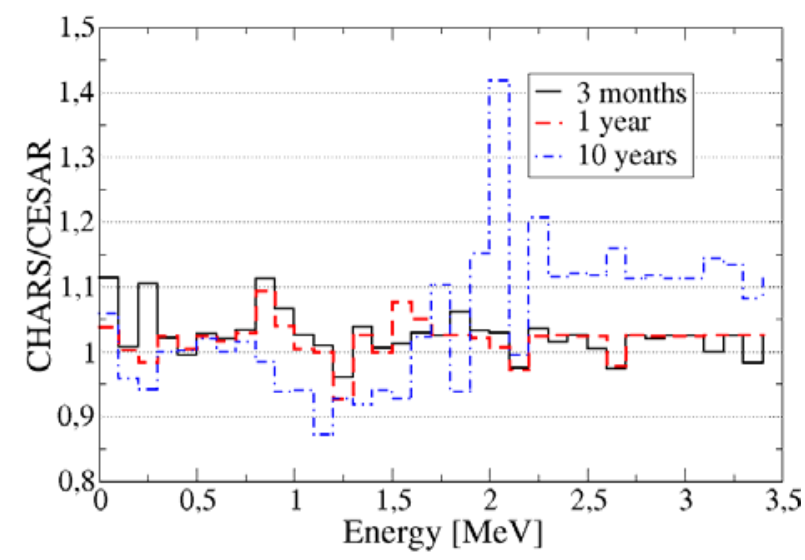

Figure 1. Ratio of spectra calculated by CESAR and CHARS for several cooling time.

Figure 1 shows good agreement between spectra calculated by these two codes for 3 months and 1 year of cooling. The two codes use the same database for the neutron transport part and fission yield (JEFF3.1) but use different ones for nuclear structure data: ENSDF for CHARS (except for ${ }^{106} \mathrm{Rh}$ which came from JEFF3.1) and JEFF3.1 for CESAR. The agreement is very good regarding the total gamma emission on a wide range of cooling time (Table 1). The discrepancy at 10 years for energies above $2 \mathrm{MeV}$ comes from a greater production of ${ }^{106} \mathrm{Rh}$ with MURE and the contribution of this nucleus in the spectrum above $2 \mathrm{MeV}$ increases with time. In addition CESAR does not consider the nuclei ${ }^{102} \mathrm{Rh}$ and ${ }^{208} \mathrm{Tl}$. This leads to the peaks in energy range $2-2.1 \mathrm{MeV}$, 2.2-2.3 $\mathrm{MeV}$ and 2.6-2.7 MeV.

Table 1. Gamma emission of a UOx spent fuel.

\begin{tabular}{ccc}
\hline $\begin{array}{c}\text { Cooling } \\
\text { time }\end{array}$ & $\begin{array}{c}\text { CESAR } \\
\left(\gamma \cdot \mathrm{s}^{-1} \cdot \mathrm{tHM}^{-1}\right)\end{array}$ & $\begin{array}{c}\text { CHARS } \\
\left(\gamma \cdot \mathrm{s}^{-1} \cdot \mathrm{tHM}^{-1}\right)\end{array}$ \\
\hline 3 months & $1.11 \times 10^{17}$ & $1.14 \times 10^{17}$ \\
1 year & $3.44 \times 10^{16}$ & $3.52 \times 10^{16}$ \\
10 years & $5.59 \times 10^{15}$ & $5.58 \times 10^{15}$ \\
100 years & $6.21 \times 10^{14}$ & $6.30 \times 10^{14}$ \\
\hline
\end{tabular}




\subsubsection{Neutron Spectra of $\mathrm{PuO}_{2}$}

In the work of E.F. Shores [10] a benchmark of the SOURCES code was performed using neutron spectra measurements of several plutonium oxides of various compositions. This benchmark was also used to validate CHARS. Results of neutron yields are presented in Table 2. References about measurements are available in [10].

Table 2. Measured and calculated neutron source magnitudes.

\begin{tabular}{cccc}
\hline Sample & $\begin{array}{c}\text { Measured } \\
{\left[\mathrm{n} . \mathrm{s}^{-1}\right]}\end{array}$ & $\begin{array}{c}\text { CHARS } \\
{\left[{\left.\mathrm{n} . \mathrm{s}^{-1}\right]}\right.}\end{array}$ & $\begin{array}{c}\text { Difference } \\
{[\%]}\end{array}$ \\
\hline JAERI1 & $184.7 \pm 4.8 \mathrm{sf}$ & 197 & 6.41 \\
& $157.8 \pm 9.8(\alpha, \mathrm{n})$ & 125 & -20.9 \\
& $342.5 \pm 8.6$ total & 321 & -6.19 \\
JAERI2 & $42.1 \pm 1.1 \mathrm{sf}$ & 42 & -0.19 \\
& $51.3 \pm(\alpha, \mathrm{n})$ & 47 & -7.60 \\
ORNL & $93.4+/-2.3$ total & 89 & -4.66 \\
SRS & $8.55 \times 10^{4} \pm 867$ & $7.63 \times 10^{4}$ & -12.0 \\
& $\pm 1.28 \times 10^{4}$ & $8.62 \times 10^{5}$ & 0.87 \\
PNL & $2.3 \times 10^{5}$ & $2.35 \times 10^{5}$ & 2.01 \\
\hline
\end{tabular}

Table 2 shows an acceptable agreement between CHARS and the measurements with a discrepancy between $-12 \%$ and $6.41 \%$ for the total yield. These differences are mainly due to the $(\alpha, n)$ part where the discrepancy can be high (-20.9\% for the JAERI1 sample). Shapes of the spectra are in good agreement with those calculated by SOURCES as shown in Figure 2.

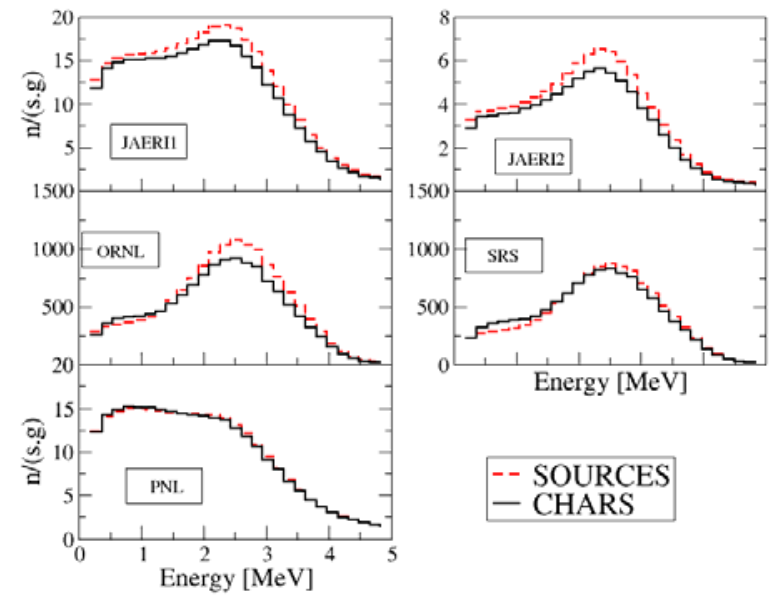

Figure 2. Neutron spectra calculated by CHARS and SOURCES for one gram of samples of plutonium dioxide.

\section{Application}

In order to save the fissionable natural resources, the use of the thorium cycle is a good candidate for replacing the current $\mathrm{U} / \mathrm{Pu}$ cycle since the fissile nucleus of the cycle, ${ }^{233} \mathrm{U}$, has neutronic properties favorable to a much better regeneration of fissile material in thermal reactors. Moreover, as the nuclei of the thorium cycle are lighter than in the $\mathrm{U} / \mathrm{Pu}$ cycle, the production of minor actinides is significantly reduced and hence waste inventories could be much lower. However, the use of Th is only viable if the spent fuel is reprocessed to recover the fissile ${ }^{233} \mathrm{U}$, which does not exist in nature. This reprocessing will involve a heavy industrial infrastructure, particularly since thorium based spent fuel contains small quantities of ${ }^{232} \mathrm{U}$, which is the mother of the hard gamma emitter $\left({ }^{208} \mathrm{Tl}\right)$ of $2.6 \mathrm{MeV}$. This gamma activity will certainly be a problem for the shielding of the back end fuel cycle especially for the manufacturing of new $\mathrm{Th} / \mathrm{U}$ mixed oxide (MOX) fuel since the ${ }^{232} \mathrm{U}$ (half life of 69 years) is present in the spent fuel uranium vector.

At present MOX fabrication plants such as MELOX in France use the MIMAS process for $\mathrm{Pu} / \mathrm{U}$ based $\mathrm{MOX}$ fuels. This consists of two stages of blending. In the first one, called the primary blend, $\mathrm{PuO}_{2}$ powder and $\mathrm{UO}_{2}$ powder are blended together. At this point the amount of $\mathrm{PuO}_{2}$ is 30 weight \%. In the second stage, $\mathrm{UO}_{2}$ is added to the mix in order to achieve the desired plutonium content. The secondary blend is then pelletized and sintered before being inserted into fuel rods and then fuel assemblies. The goal of this study is to show the impact on radiation exposure of the fabrication of ThU MOX fuel comparatively to the conventional UPu MOX fuel.

\subsection{Problem definition}

Due to its high radiation level the first stage of blending has been chosen for the comparison. The plutonium used for the MOX came from a PWR 5\% ${ }^{235} \mathrm{U}$ at $55 \mathrm{GWd} / \mathrm{tHM}$ with 5 years of cooling before reprocessing and 6 years of cooling after. This elapsed time corresponds to an ${ }^{241} \mathrm{Am}$ content of $3 \%$. The uranium for the ThU fuel came from a PWR loaded with $\mathrm{ThPuO}_{2}$, $11 \%$ of $\mathrm{Pu}$ at $55 \mathrm{GWd} / \mathrm{tHM}$ with 5 years of cooling before reprocessing; several different cooling times after reprocessing are used in order to see the impact of the cooling time on the dose rate.

The design of the first blending workshop consists of a stainless steel glove box of thickness $1 \mathrm{~cm}$ with a lead glass window of $2 \mathrm{~cm}$ in thickness. The mixture, which is $60 \mathrm{~kg}$ of MOX or $\mathrm{ThUO}_{2}$ is in a stainless steel bottle of $2 \mathrm{~mm}$ in thickness surrounded by a neutron protection layer of $10 \mathrm{~cm}$ thick polyethylene. The dose rate is estimated at $10 \mathrm{~cm}$ from the surface of glove-box window using MCNP5 and the track-length estimator (F4 tally) modified by the AP fluence-to-dose conversion factor from ICRP [11].

\subsection{Source calculations}

Given the composition of the first blend, CHARS was used to calculate both gamma and neutron spectra. The composition assumed two oxygen atoms for each heavy nucleus. The thorium used for the $\mathrm{ThUO}_{2}$ powder of 30 weight $\%$ of $\mathrm{UO}_{2}$ was pure ${ }^{232} \mathrm{Th}$, and depleted uranium $\left(2.01 \%\right.$ mol. $\left.{ }^{235} \mathrm{U}\right)$ was used for the MOX powder containing 30 weight $\%$ of $\mathrm{PuO}_{2}$. Results of this calculation are shown in Figure 3 for gamma spectra. The origin of gammas and neutrons for the $\mathrm{ThUO}_{2}$ powder is the ${ }^{232} \mathrm{U}$ decay chain while for the MOX case is mainly ${ }^{238} \mathrm{Pu}$ and ${ }^{240} \mathrm{Pu}$ for neutrons and ${ }^{241} \mathrm{Am}$ for gammas. Total 
neutron emission is lower in the $\mathrm{ThUO}_{2}$ case but the spectrum is slightly harder due to the highest energy of alphas (up to $8.78 \mathrm{MeV}$ (from ${ }^{212} \mathrm{Po}$ ) whereas the maximum energy for the $\mathrm{UPuO}_{2}$ standard MOX case is $5.5 \mathrm{MeV}\left(\right.$ from $\left.{ }^{238} \mathrm{Pu}\right)$ ).

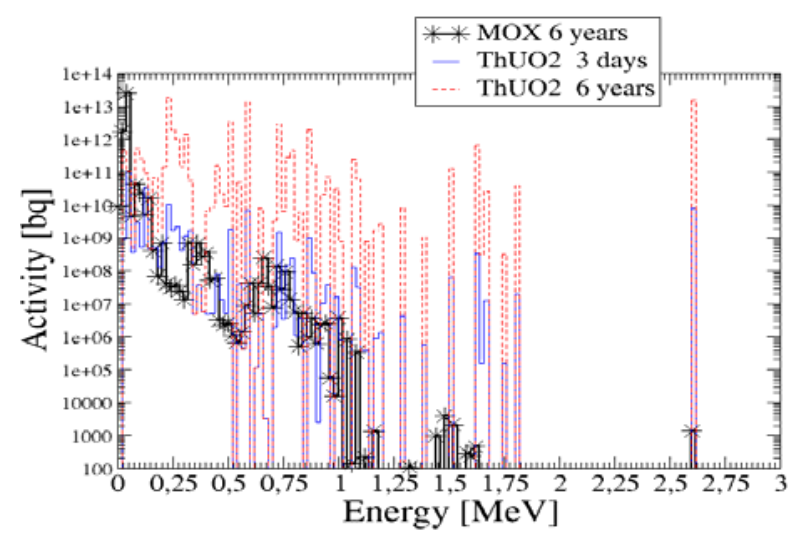

Figure 3. Gamma Spectra of the two different powders.

\subsection{Results}

Table 3 clearly shows the problem caused by the ${ }^{232} U$ in the fabrication of ThU fuels. It presents the dose rate behind the glove-box when using $\mathrm{ThUO}_{2}$ powder of several cooling times relative to the dose rate calculated for the reference MOX powder after 6 years of cooling; i.e $\mathrm{ThUO}_{2}$ dose rate / $\mathrm{MOX}$ dose rate. It appears that even if $\mathrm{ThUO}_{2}$ fuel is made 3 days after reprocessing the dose rate is still 8 times higher than in the reference case. In the worst case: when the quantity of ${ }^{208} \mathrm{Tl}$ is at its maximum, the dose rate is 16500 times higher. On the other hand, the neutron dose rate is reduced and a lighter neutron shielding can be used.

Table 3. Relative dose rate $\left(\mathrm{ThUO}_{2} / \mathrm{MOX}\right)$.

\begin{tabular}{cccc}
\hline $\begin{array}{c}\text { Time after } \\
\text { reprocessing }\end{array}$ & $\begin{array}{c}\text { Gamma } \\
\text { (Secondary } \gamma)\end{array}$ & Neutron & Total \\
\hline 3 days & $149(0.083)$ & 0.076 & 8 \\
1 months & $9990(0.103)$ & 0.089 & 536 \\
1 year & $1.02 \times 10^{5}(0.237)$ & 0.208 & 5470 \\
6 years & $2.9 \times 10^{5}(0.51)$ & 0.447 & 15600 \\
10 years & $3.08 \times 10^{5}(0.53)$ & 0.467 & 16500 \\
\hline
\end{tabular}

In the next calculation, the cooling time is kept to 10 years (worst case) and the lead glass thickness is increased. The results are presented in Table 4. In order to obtain the same dose rate as the reference case the lead glass window must be $43.5 \mathrm{~cm}$, which is not realistic.

Table 4. Relative dose rate versus lead glass thickness.

\begin{tabular}{lcccccc}
\hline Thickness $(\mathrm{cm})$ & 2 & 5 & 10 & 20 & 30 & 43.5 \\
\hline $\begin{array}{l}\text { Relative } \\
\text { dose rate }\end{array}$ & 16500 & 8330 & 2700 & 266 & 25 & 1 \\
\hline
\end{tabular}

\section{Conclusion}

A set of tools to characterize spent fuel has been developed and its reliability has been shown with several benchmarks. CHARS will be available at the OECD NEA at the end of 2013 at the time of the update of the MURE package. With this tool and MCNP, we demonstrate in a qualitative way that the fabrication of $\mathrm{ThUO}_{2}$ fuel is not possible on an industrial scale using currently used processes such as MIMAS in glove-boxes. Complete automation of the fuel re-fabrication process may be required which could place severe problems for long-term maintenance of the facility. Research into other fabrication processes is clearly needed (e.g. impregnation of low density $\mathrm{ThO}_{2}$ pellets with uranyl nitrate [12]).

\section{References}

[1] O. Méplan et al., MURE: MCNP Utility for Reactor Evolution description of the methods, first applications and results, Proc. Int. European Nuclear Conference (ENC05), Versailles, France, dec. 14-18, (2005), pp. 1-7.

[2] F.B. Brown, MCNP - A general Monte Carlo $n$ particle Transport Code, LANL report 12625-M, Los Alamos National Laboratory, (1997).

[3] J.K. Tuli, Evaluated nuclear structure data file: a manual for preparation of data sets, BNL-NCS-51655-01/02 Rev., Brookhaven National Laboratory, (2001).

[4] LBNL Isotopes project, evaluated nuclear structure data file (ENSDF). http://ie.lbl.gov/databases/ensdfserve.html

[5] E.F. Shores, Data updates for the SOURCES-4A computer code, Nuclear Instruments and Methods in Physics Research B, 179, (2001), pp. 78-82.

[6] J. F. Ziegler et al., SRIM - The stopping and range of ions in matter, Nuclear Instruments and Methods in Physics Research B, 268, (2010), pp. 1818-1823.

[7] T. Murata et al., Evaluation of the $(\alpha, x n)$ reaction data for JENDL/AN-2005, JAEA-Research 2006-052, Japan Atomic Energy Agency, (2006).

[8] A. J. Koning et al., TALYS-1.0. Proc. Int. Conf. on nuclear data for science and technology, Nice, France, april 22-27, (2007), pp. 211-214.

[9] J.M. Vidal, et. al., CESAR: A code for nuclear fuel and waste characterization, WM'06 Conference, Tucson, US, feb. 26 mar. 2, (2006).

[10] E.F. Shores, Plutonium oxide benchmark problems for the SOURCES code, Applied Radiation and Isotopes, 62 (5), (2005), pp. 699-704.

[11] ICRP, Conversion coefficients for radiological protection quantities for external radiation exposures, ICRP Publication 116, Ann. ICRP 40(2-5), (2010).

[12] IAEA, Thorium fuel cycle-potential benefits and challenges, IAEA TECDOC 1450, IAEA, (2005). 